\title{
A Note on Spurious Regressions between Stationary Series
}

\author{
Jen-Je Su* \\ Department of Applied and International Economics \\ Massey University
}

This version: February 8, 2006

Running Title: Spurious Regression, Convergent t-Statistic

\begin{abstract}
This paper examines if the convergent t-test suggested by Sun (2004) is able to solve spurious regressions with stationary series. In brief, we find that the convergent t-test does provide better control over size compared to the usual t-test and its Newey-West modification and, in most cases implementing a pre-whiting procedure size is further controlled.
\end{abstract}

* Jen-Je Su

Applied and International Economics Department

Massey University

Private Bag 11222

Palmerston North, New Zealand

Email: j.j.su@massey.ac.nz

Tel: 64-6-3505799 ext 2666

Fax: 64-6-3505660 


\section{Introduction}

It is a well-known fact that the use of nonstationary data can lead to spurious regressions. Granger and Newbold (1974) first found via Monte Carlo simulations that, given two independent random walks, it is likely that a regression of one on the other will produce a "significant" slope coefficient according to the usual t-test. Since the two walks are actually unrelated the

statistical significance is spurious and misleading. Later, Phillips (1986) developed an asymptotic theory for regression between two unrelated I(1) processes, showing that the usual t-statistic does not have a limiting distribution but diverges as the sample size goes to infinity.

Recently, Sun (2004) found that the seemingly inevitable divergent behaviour of the usual t-test is due to the use of a standard error that underestimates the true variation of the slope estimator. He showed that once an appropriate standard error estimate, such as the one suggested in Kiefer and Vogelsang (2002; hereafter, KV), is used, the resulting t-statistic is no longer divergent.

There is also interest in studying spurious regressions in a stationary environment. As a matter of fact, Granger, Hyung and Jeon (2001; hereafter, GHJ) have found that a spurious regression can even occur in an occasion that the usual t-statistic is actually convergent. Using extensive finite-sample simulations, GHJ gave evidence that the null hypothesis of zero-slope in a regression with two 
independent stationary autoregressive series or medium-to-long moving averages is severely over-rejected.

In this paper, we are interested in the performance of the convergent tstatistic of KV (2002) and Sun (2004) in the situations considered in GHJ (2001) through Monte-Carlo simulations. In brief, we find that the convergent t-test provides much better control over size than the usual t-test (and its Newey-West modification). In most cases, although the convergent t-test does not produce correct size, the situation is not too bad - particularly, when the sample size is large. However, there are occasions that the likelihood of over-rejection is still high. We also find that implementing an $\mathrm{AR}(1)$ pre-whitening procedure may produce further control over size, but with some exceptions.

The paper is organised as follows. Section 2 provides a short review regarding HAC robust tests and spurious regressions. Section 3 reports and discusses our simulation results. Finally, Section 4 concludes.

\section{HAC Robust Test Statistics}

Consider the regression

$$
Y_{t}=\alpha+\beta X_{t}+u_{t}, \mathrm{t}=1,2, \ldots, \mathrm{T} \text {. }
$$

Let $\hat{\beta}$ be the OLS estimate for $\beta$ and $t_{\beta}$ be the corresponding t-ratio. It is well known that when the error term in (1) is heteroskedastic or autocorrelated $t_{\beta}$ is not asymptotically valid. Alternatively, a kernel-based HAC robust t-statistic can be defined as follows: 


$$
t^{*}=\frac{\hat{\beta}}{\hat{\sigma}_{\hat{\beta}}^{2}}=\frac{\hat{\beta}}{\left[T \hat{\Omega}_{M} /\left(\sum_{t=1}^{T}\left(X_{t}-\bar{X}\right)^{2}\right)^{2}\right]^{1 / 2}},
$$

where

$$
\begin{gathered}
\hat{\sigma}_{\hat{\beta}}^{2}=\frac{T \hat{\Omega}_{M}}{\left(\sum_{t=1}^{T}\left(X_{t}-\bar{X}\right)^{2}\right)^{2}}, \\
\hat{\Omega}_{M}=\frac{1}{T} \sum_{j=-(T-1)}^{T-1} \kappa(j / M) \hat{\Gamma}(j), \\
\hat{\Gamma}(j)=\sum_{t=j+1}^{T}\left(X_{t}-\bar{X}\right)^{2} \hat{u}_{t} \hat{u}_{t-j} \text { for } j \geq 0, \hat{\Gamma}(j)=\hat{\Gamma}(-j) \text { for } j<0 .
\end{gathered}
$$

Here, $M$ is the bandwidth parameter and $\kappa(x)$ is a kernel function. Following Newey and West (1987), we consider the Bartlett kernel, $\kappa(x)=(1-|x|)^{-1}$ if $|x|<1$ and 0 otherwise, throughout paper.

To ensure that $\hat{\sigma}_{\hat{\beta}}^{2}$ is a consistent estimate for the variance of $\hat{\beta}$, the conventional approach requires that " $M, T \rightarrow \infty$ and $M=o(T)$." See Newey and West (1987) for an early development and den Haan and Levin (1997) for a recent review. Alternatively, Kiefer and Vogelsang (2002) suggest using $M=T$ (the full bandwidth) in the calculation of $\hat{\Omega}_{M}$ (denoted by $\left.\hat{\Omega}_{M=T}\right)^{1}$. This rate for $M$ clearly violates the $M=o(T)$ rule and $\hat{\Omega}_{M=T}$ is not consistent. However, as shown in Kiefer and Vogelsang (2002), the test statistic using $\hat{\Omega}_{M=T}$ is asymptotically pivotal and exhibits a better finite-sample size control than the conventional

\footnotetext{
${ }^{1}$ Recently, Kiefer and Vogelsang (2005) suggest using $M=b T$, where $b \in(0,1]$ is a fixed number. An obvious trade-off has been found: a small bandwidth (small b) leads to tests with higher power but greater size distortions and a large bandwidth (large b) leads to tests with lower power but less size distortions. Since controlling for size distortions is our main concern, we stick to the choice of "M=T" (i.e. b=1).
} 
approach. In this paper, we will refer the HAC robust t-test assuming “ $M, T \rightarrow \infty$ and $M=o(T)$ ” as $t_{N W}^{*}$ and the test using “ $M=T$ ” as $t_{K V}^{*}$.

\section{Monte Carlo Simulations}

In this section we report the results of Monte Carlo experiments designed to investigate finite-sample size of the three previously discussed tests - namely, t, $t_{N W}^{*}$ and $t_{K V}^{*}$. The bandwidth parameter (M) in $t_{N W}^{*}$ is set equal to the integer part of $4(T / 100)^{1 / 4}$, as in GHJ (2001). Also, an AR(1) pre-whitening procedure of Andrew and Monahan (1992) is implemented for $t_{N W}^{*}$ and $t_{K V}^{*}-$ denoted by $t_{N W, P W}^{*}$ $t_{N W, P W}^{*}$ respectively. All simulations are preformed in GAUSS and the results (the rejection rates) are calculated by comparing critical values at 5\% (that is, 1.96 for the usual t-test and the NW test and 3.76 for the KV test) via 5,000 iterations. In the following, simulation results of two cases that spurious regressions between two stationary series found by GHJ (2001).

\section{CASE I: Spurious regression between AR processes}

The first experiment is done by considering $Y_{t}$ and $X_{t}$ in (1) defined by independent $\mathrm{AR}(1)$ processes:

$$
Y_{t}=\phi_{y} Y_{t-1}+\varepsilon_{y, t} \text { and } X_{t}=\phi_{x} X_{t-1}+\varepsilon_{x, t},
$$


where $\varepsilon_{y, t}, \varepsilon_{x, t}$ are each i.i.d. $N(0,1)$, independent to one another. Simulations results at sample size $\mathrm{T}=100,250,500,1000$, and 2000 are reported in Tables 1 for $\phi_{x}=\phi_{y}=\phi$ and Table 2 for $\phi_{x} \neq \phi_{y}$. These results correspond to those reported in Tables 1 and 2 of GHJ (2001).

The results in Table 1 can be summarized as follows. Spurious rejections appear frequently if the usual t-test is used and the test is evidently divergent as $\mathrm{T}$ increases for $\phi=0.99$ or larger. The Newey-West method is helpful; but its advantage is less impressive if $\phi$ is close to 1.0 and T is large. Also, its divergent behaviour becomes apparent for $\phi=1.0$ but at a slower rate, as predicted in Phillips (1998). These results are generally in agreement with those found in GHJ (2001). Performance of the KV test is quite impressive compared to the other two tests - the size is accurate for $\phi=0.90$ or less and still under control even for $\phi=0.99$ if the sample size is large. Moreover, the test is convergent for random walks ( $\phi=1.0$ ), which is in accordance to the asymptotic theory of Sun (2004). However, the frequency of rejection is still much higher than the nominal size when $\phi>0.95$. In the random walk case, the size of the KV test reaches 0.33 regardless the sample size.

Implementing a pre-whiting procedure does help for the NW test in taming size. Taking $\phi=0.9$ and $\mathrm{T}=500$ for example, the rejection rate is reduced dramatically from 0.224 to 0.091 . Even so, the procedure fails to make the NW test convergent when $\phi$ is close to 1 . When the KV test is considered, a prewhiting procedure can successfully reduce size distortion, provided that the 
autoregression parameter $\phi$ is no larger than 0.95 . Taking $\phi=0.95$ and $\mathrm{T}=500$ for example, the rejection rate is reduced from 0.098 to 0.076 , which is close to the nominal size. However, for $\phi=0.99$ or larger, the procedure actually inflates the rejection rate and makes the test divergent when $\mathrm{T}$ is large. For example, at $\mathrm{T}=2000$, the rejection rate increases from 0.151 to 0.285 and from 0.326 to 0.667 for $\phi$ equals 0.995 and 1.0, respectively. Therefore, a warning should be addressed in implementing a pre-whitening procedure.

Table 2 generally agrees with Table 1 . The KV test is obviously the best. Performing a pre-whiting procedure is a plus in all cases. Also, as shown in GHJ (2001), rejection rates are similar for $\left(\phi_{x}=a, \phi_{y}=b\right)$ and $\left(\phi_{x}=b, \phi_{y}=a\right)$ if $a \neq b$ according to the usual t-test and the NW test. Such a symmetry does not appear for the KV test: rejection rates for $\left(\phi_{x}=a, \phi_{y}=b\right)$ tend to be larger than those for $\left(\phi_{x}=a, \phi_{y}=b\right)$ if $a>b$.

\section{CASE II: Spurious regression between MA processes}

The second experiment assumes that $Y_{t}$ and $X_{t}$ are generated by two independent $\mathrm{MA}(\mathrm{K})$ processes:

$$
Y_{t}=\sum_{j=0}^{K} \varepsilon_{y, t-j} \text { and } X_{t}=\sum_{j=0}^{K} \varepsilon_{x, t-j}
$$

where $\varepsilon_{x t}, \varepsilon_{y t}$ are each i.i.d. $N(0,1)$. Table 3 reports the simulation results for $\mathrm{K}$ taking the values 5, 10, 20, 50, or 75 and sample size varying from 100 to 2000 . The results correspond to those reported in Tables 4 of GHJ (2001). 
The results are generally consistent with those of the previous case. The usual t-test over-rejects frequently, almost one-half for $\mathrm{K}=10$; the rejection frequency increases steadily with $\mathrm{K}$, but is relatively stable over T. Frequency of over-rejection is greatly reduced using the NW test, and is reduced further when the $\mathrm{KV}$ test is used. Taking $\mathrm{T}=250$ and $\mathrm{K}=10$ for example, rejection rates of the usual t-test, the NW test and the KV test are $0.491,0.200$ and 0.082 , respectively. For both HAC robust tests, rejection frequency increases steadily with $\mathrm{K}$ when $\mathrm{T}$ is fixed but decreases with $\mathrm{T}$ when $\mathrm{K}$ is fixed. Also, in all cases studied, size distortion is reduced further if a pre-whitening procedure is performed. Consider the $\mathrm{KV}$ test with pre-whitening, the rejection rate of $\mathrm{K}=75$ is around 0.1 at $\mathrm{T}=500$ and less than 0.1 at $\mathrm{T}>500$. The pre-whitened NW test seems to be very conservative when $\mathrm{K}$ is small and $\mathrm{T}$ is large - the null hypothesis tends to be under-rejected.

\section{Conclusion}

This paper assesses whether the convergent t-test of KV (2002) and Sun (2004) is able to solve spurious regressions found in GHJ (2001). To do so, intensive Monte-Carlo experiments have been conducted. In brief, it has been found that the convergent t-test delivers much better control over size comparing to the usual t-statistic and its Newey-West modification. Also, for most cases studied, implementing the convergent t-test with an AR(1) pre-whiting procedure produced further control over size. Yet, in many occasions, the rate of bias 
rejection is still much higher than the nominal size when the convergent test is used.

\section{References}

Andrews, D.W.K. and J.C. Monahan (1992) An improved heteroskedasticity and autocorrelation consistent covariance estimator, Econometrica, 59, 953-966.

den Haan W.J. and A. Levin (1997) A practitioner's guide to robust covariance matrix estimation, in G. Maddala and C. Rao (eds), Handbook of Statistics: Robust Inference, Volume 15, Elsevier, New York, 291-341.

Granger, C.W.J. and P. Newbold (1974) Spurious regressions in econometrics, Journal of Econometrics, 2, 111-120.

Granger, C.W.J., N. Hyung and Y, Jeon (2001) Spurious regressions with stationary series, Applied Economics, 33, 899-904.

Kiefer, N.M. and T.J. Vogelsang (2002) Heteroskedasticity-autocorrelation robust testing using bandwidth equal to sample size, Econometric Theory, 18, 13501366.

Kiefer, N.M. and T.J. Vogelsang (2005) A new asymptotic theory for heteroskedasticity-autocorrelation robust tests, Econometric Theory, 21, 11301164.

Newey, W.K. and K.D. West (1987) A simple, positive semi-definite, heteroskedasticity and autocorrelation consistent covariance matrix, Econometrica, 55, 703-708.

Phillips, P.C.B. (1986) Understanding spurious regressions in econometrics, Journal of Econometrics, 33, 311-340.

Phillips, P.C.B. (1998) New toots for understanding spurious regressions, Econometrica, 66, 1299-1325.

Sun Y. (2004) A convergent t-statistic in spurious regressions, Econometric Theory, 20, 943-962 
Table 1: Spurious regression between AR processes $\left(\phi_{x}=\phi_{y}=\phi\right)$

\begin{tabular}{|c|c|c|c|c|c|c|}
\hline$\phi$ & $\mathrm{T}$ & $t$ & $t_{N W}^{*}$ & $t_{N W, P W}^{*}$ & $t_{K V}^{*}$ & $t_{K V, P W}^{*}$ \\
\hline \multirow{5}{*}{0.50} & 100 & .138 & .110 & .096 & .069 & .063 \\
\hline & 250 & .135 & .079 & .068 & .051 & .047 \\
\hline & 500 & .135 & .073 & .056 & .051 & .050 \\
\hline & 1000 & .126 & .065 & .055 & .050 & .049 \\
\hline & 2000 & .131 & .059 & .052 & .050 & .048 \\
\hline \multirow{5}{*}{0.75} & 100 & .292 & .174 & .125 & .096 & .077 \\
\hline & 250 & .289 & .123 & .079 & .064 & .057 \\
\hline & 500 & .308 & .114 & .073 & .061 & .058 \\
\hline & 1000 & .300 & .086 & .054 & .058 & .056 \\
\hline & 2000 & .300 & .083 & .053 & .052 & .050 \\
\hline \multirow{5}{*}{0.9} & 100 & .522 & .312 & .178 & .140 & .106 \\
\hline & 250 & .523 & .243 & .115 & .094 & .077 \\
\hline & 500 & .525 & .224 & .091 & .073 & .063 \\
\hline & 1000 & .525 & .168 & .072 & .060 & .056 \\
\hline & 2000 & .524 & .151 & .058 & .058 & .056 \\
\hline \multirow{5}{*}{0.95} & 100 & .616 & .412 & .230 & .199 & .149 \\
\hline & 250 & .653 & .381 & .162 & .136 & .103 \\
\hline & 500 & .659 & .362 & .118 & .098 & .076 \\
\hline & 1000 & .662 & .305 & .091 & .077 & .067 \\
\hline & 2000 & .663 & .267 & .073 & .066 & .059 \\
\hline \multirow{5}{*}{0.99} & 100 & .739 & .557 & .334 & .299 & .207 \\
\hline & 250 & .804 & .612 & .288 & .252 & .170 \\
\hline & 500 & .822 & .625 & .236 & .197 & .145 \\
\hline & 1000 & .832 & .597 & .188 & .138 & .114 \\
\hline & 2000 & .839 & .578 & .174 & .116 & .121 \\
\hline \multirow{5}{*}{0.995} & 100 & .741 & .573 & .343 & .308 & .213 \\
\hline & 250 & .827 & .648 & .325 & .295 & .200 \\
\hline & 500 & .856 & .697 & .301 & .250 & .181 \\
\hline & 1000 & .879 & .695 & .336 & .205 & .200 \\
\hline & 2000 & .880 & .687 & .407 & .151 & .285 \\
\hline \multirow{5}{*}{1.0} & 100 & .769 & .613 & .372 & .339 & .231 \\
\hline & 250 & .856 & 699 & .382 & .327 & .222 \\
\hline & 500 & .887 & .772 & .439 & .328 & .253 \\
\hline & 1000 & .923 & .810 & .624 & .334 & .435 \\
\hline & 2000 & .942 & .849 & .793 & .326 & .667 \\
\hline
\end{tabular}


Table 2: Spurious regression between two AR processes $\left(\phi_{\mathrm{x}} \neq \phi_{\mathrm{y}}\right)$

\begin{tabular}{|c|c|c|c|c|c|c|}
\hline$\left(\phi_{\mathrm{y}}, \phi_{\mathrm{x}}\right)$ & $\mathrm{T}$ & $t$ & $t_{N W}^{*}$ & $t_{N W, P W}^{*}$ & $t_{K V}^{*}$ & $t_{K V, P W}^{*}$ \\
\hline \multirow{3}{*}{$(0.75,0.90)$} & 100 & .387 & .217 & .132 & .107 & .088 \\
\hline & 500 & .382 & .137 & .069 & .065 & .059 \\
\hline & 1000 & .399 & .112 & .060 & .057 & .052 \\
\hline \multirow{3}{*}{$(0.90,0.75)$} & 100 & .379 & .218 & .140 & .102 & .083 \\
\hline & 500 & .391 & .149 & .075 & .063 & .057 \\
\hline & 1000 & .387 & .111 & .061 & .055 & .051 \\
\hline \multirow{3}{*}{$(0.75,0.95)$} & 100 & .401 & .219 & .141 & .120 & .091 \\
\hline & 500 & .427 & .159 & .073 & .077 & .069 \\
\hline & 1000 & .417 & .127 & .067 & .069 & .064 \\
\hline \multirow{3}{*}{$(0.95,0.75)$} & 100 & .406 & .230 & .147 & .100 & .078 \\
\hline & 500 & .423 & .153 & .070 & .064 & .056 \\
\hline & 1000 & .418 & .121 & .058 & .053 & .048 \\
\hline \multirow{3}{*}{$(0.75,1.0)$} & 100 & .443 & .237 & .142 & .133 & .092 \\
\hline & 500 & .448 & .174 & .075 & .099 & .081 \\
\hline & 1000 & .464 & .140 & .064 & .089 & .080 \\
\hline \multirow{3}{*}{$(1.0,0.75)$} & 100 & .444 & .243 & .156 & .100 & .076 \\
\hline & 500 & .452 & .167 & .077 & .053 & .040 \\
\hline & 1000 & .467 & .139 & .066 & .066 & .060 \\
\hline \multirow{3}{*}{$(0.90,0.95)$} & 100 & .553 & .337 & .194 & .171 & .122 \\
\hline & 500 & .576 & .263 & .090 & .084 & .069 \\
\hline & 1000 & .576 & .226 & .074 & .066 & .060 \\
\hline \multirow{3}{*}{$(0.95,0.90)$} & 100 & .558 & .344 & .192 & .165 & .118 \\
\hline & 500 & .585 & .276 & .094 & .081 & .066 \\
\hline & 1000 & .591 & .223 & .080 & .063 & .057 \\
\hline \multirow{3}{*}{$(0.9,1.0)$} & 100 & .611 & .394 & .206 & .189 & .128 \\
\hline & 500 & .648 & .346 & .107 & .122 & .089 \\
\hline & 1000 & .651 & .278 & .080 & .098 & .079 \\
\hline \multirow{3}{*}{$(1.0,0.9)$} & 100 & .608 & .406 & .245 & .178 & .134 \\
\hline & 500 & .647 & .348 & .117 & .082 & .063 \\
\hline & 1000 & .649 & .280 & .086 & .060 & .048 \\
\hline \multirow{3}{*}{$(0.95,1.0)$} & 100 & .687 & .495 & .266 & .243 & .157 \\
\hline & 500 & .731 & .469 & .144 & .146 & .099 \\
\hline & 1000 & .747 & .424 & .099 & .113 & .084 \\
\hline \multirow{3}{*}{$(1.0,0.95)$} & 100 & .670 & .484 & .295 & .241 & .172 \\
\hline & 500 & .735 & .481 & .172 & .120 & .089 \\
\hline & 1000 & .747 & .418 & .121 & .085 & .063 \\
\hline
\end{tabular}


Table 3: Spurious Regression between MA(k) processes

\begin{tabular}{|c|c|c|c|c|c|c|}
\hline K & $\mathrm{T}$ & $t$ & $t_{N W}^{*}$ & $t_{N W, P W}^{*}$ & $t_{K V}^{*}$ & $t_{K V, P W}^{*}$ \\
\hline \multirow{5}{*}{2} & 100 & .176 & .103 & .073 & .062 & .051 \\
\hline & 250 & .178 & .081 & .056 & .058 & .051 \\
\hline & 500 & .179 & .072 & .048 & .054 & .048 \\
\hline & 1000 & .178 & .066 & .048 & .051 & .050 \\
\hline & 2000 & .179 & .063 & .045 & .050 & .049 \\
\hline \multirow{5}{*}{5} & 100 & .339 & .165 & .087 & .084 & .060 \\
\hline & 250 & .330 & .118 & .046 & .063 & .050 \\
\hline & 500 & .338 & .113 & .037 & .059 & .051 \\
\hline & 1000 & .325 & .085 & .034 & .052 & .047 \\
\hline & 2000 & .333 & .083 & .035 & .052 & .051 \\
\hline \multirow{5}{*}{10} & 100 & .490 & .269 & .129 & .122 & .083 \\
\hline & 250 & .491 & .200 & .070 & .082 & .062 \\
\hline & 500 & .478 & .166 & .037 & .060 & .048 \\
\hline & 1000 & .462 & .132 & .029 & .060 & .053 \\
\hline & 2000 & .470 & .104 & .022 & .048 & .045 \\
\hline \multirow{5}{*}{15} & 100 & .579 & .359 & .172 & .156 & .104 \\
\hline & 250 & .569 & .267 & .081 & .094 & .066 \\
\hline & 500 & .561 & .227 & .046 & .069 & .054 \\
\hline & 1000 & .559 & .174 & .036 & .060 & .052 \\
\hline & 2000 & .557 & .150 & .028 & .060 & .053 \\
\hline \multirow{5}{*}{20} & 100 & .637 & .420 & .217 & .193 & .126 \\
\hline & 250 & .620 & .325 & .107 & .108 & .074 \\
\hline & 500 & .607 & .285 & .061 & .083 & .061 \\
\hline & 1000 & .604 & .226 & .041 & .064 & .049 \\
\hline & 2000 & .604 & .181 & .023 & .054 & .049 \\
\hline \multirow{5}{*}{50} & 100 & .752 & .585 & .352 & .327 & .232 \\
\hline & 250 & .743 & .533 & .206 & .191 & .128 \\
\hline & 500 & .747 & .483 & .126 & .121 & .080 \\
\hline & 1000 & .747 & .412 & .070 & .087 & .060 \\
\hline & 2000 & .735 & .348 & .037 & .061 & .049 \\
\hline \multirow{5}{*}{75} & 100 & .771 & .622 & .367 & .337 & .237 \\
\hline & 250 & .792 & .610 & .272 & .250 & .173 \\
\hline & 500 & .791 & .571 & . 164 & .153 & .101 \\
\hline & 1000 & .784 & .490 & .096 & .096 & .070 \\
\hline & 2000 & .779 & .456 & .060 & .072 & .060 \\
\hline
\end{tabular}

